DOI: https://doi.org/10.24297/jam.v17i0.8507

\title{
Almost Paracontact 3-Submersions
}

\author{
T.Tshikuna-Matamba
}

Département de Mathématiques, Université Pédagogique de Kananga B.P. 282-Kananga, République Démocratique du Congo.

tshikmat@gmail.com

\begin{abstract}
In this paper, we discuss some geometric properties of Riemannian submersions whose total space is an almost paracontact manifold with 3-structure. The study is focused on the transference of structures, the geometry of the fibres and sectional curvature tensor.
\end{abstract}

2010. Mathematics Subject Classification: 53C15, 53C26, 53C55.

Keywords: Riemannian submersions, almost paracontact manifolds with 3 -structure, almost hyper para-Hermitian manifolds.

\section{Introduction}

Almost paracontact submersions are Riemannian submersions whose total space is endowed with almost paracontact structure. These submersions have been introduced by Gündüzalp and Sahin [10] where the base space is also an almost paracontact manifold. Transference of the structure from the total to the base space has been examined. The structure of the fibres as that of the base space are studied in [19, 20, 21, 22].

In this paper, we consider the case where the total space is endowed with an almost paracontact 3-structure. This class of submersions are closely related to almost contact 3-submersions, initiated by B. Watson [25, 26] and extended in [18].

According to the structure of the base space, two types of submersions are studied. Considering that the base space possesses three almost paracontact structures, the following is stated.

Let $\pi: M^{4 m+3} \longrightarrow M^{4 m^{\prime}+3}$ be an almost paracontact 3-submersion of type II. If the total space is $3-$ paracosymplectic, then the fibres are hyper-para-Kähler.

This result, among others, is the way to claim that according to the theory of foliation, an almost paracontact manifold with 3-structure possesses a hyper-para-Kähler manifold as submanifold. Indeed, Proposition 3.4, states that the fibres of an almost paracontact 3-submersion of type II are almost hyper-para-Hermitian manifolds.

Concerning the $\varphi_{i}$-holomorphic sectional curvature tensor, it is shown that if the total space of an almost paracontact 3 -submersion of type I is 3-para-cosymplectic, then the $\varphi_{i}$-holomorphic curvature tensor is preserved both on the 
horizontal and vertical distributions.

Using the product of an almost hyper-para-Hermitian manifold by an almost paracontact manifold with 3-structure, we construct an example of almost paracontact 3 -submersions of type I.

\section{Preliminaries on Manifolds}

An almost quaternionic-para-Hermitian manifold is a quintuple $\left(M, g, J_{1}, J_{2}, J_{3}\right)$ where $J_{i}^{2}=\mathbb{I}$,

(a) $(M, g)$ is a Riemannian manifold;

(b) $\left(g, J_{i}\right)$ is an almost para-Hermitian structure for $i=1,2,3$;

(c) $J_{i} \circ J_{j}=-J_{j} \circ J_{i}=J_{k}$ for $i \prec j, k \neq i$ and $k \neq j$.

Each almost para-Hermitian structure $\left(g, J_{i}\right)$ defines a local para-Kähler 2-form, $\Omega_{i}$, via $\Omega_{i}(D, E)=g\left(D, J_{i} E\right)$. This leads to define a global, non degenerate 4 -form, $\Omega$, which is the fundamental form by $\Omega=\sum_{i=1}^{3} \Omega_{i} \wedge \Omega_{i}$.

Almost quaternionic-para-Hermitian manifolds are of dimension $4 \mathrm{~m}$.

Note that locally, $\left\{J_{1}, J_{2}, J_{3}\right\}$ constitutes an adaptated basis of a subbundle $\mathcal{B}$ of the endomorphism bundle $E n d T(M)$, where $T(M)$ is the tangent bundle of $M$. By an almost hyper-Hermitian manifold, one understands a quaternionicHermitian manifold with a global adaptated basis [2].

An almost paracontact structure on a differentiable manifold $M$ is a triple $(\varphi, \xi, \eta)$, where:

(i) $\xi$ is a distinguished vector field,

(ii) $\eta$ is a differential 1 -form such that $\eta(\xi)=1$,

(iii) $\varphi$ is a tensor field of type $(1,1)$ satisfying

$$
\varphi^{2} D=D-\eta(D) \xi
$$

If in addition, $\quad M$ is furnished with a Riemannian metric $g$ such that

$$
g(\varphi D, \varphi E)=-g(D, E)+\eta(D) \eta(E)
$$

then $g$ is said to be a compatible metric and $(M, g, \varphi, \xi, \eta)$ is called an almost paracontact metric manifold.

The study of almost paracontact manifolds can be found in $[6,7,8,12,13,14,23,24]$ and [28] among others.

Deshmukh and Khan [9, p.448] have constructed a properly almost paracontact 3 -structure on $\mathbb{R}^{3}$ as follows

$$
\varphi_{1}=\left(\begin{array}{ccc}
0 & 0 & 1 \\
0 & 0 & 0 \\
1 & 0 & 0
\end{array}\right), \varphi_{2}=\left(\begin{array}{ccc}
0 & 0 & 0 \\
0 & 0 & 1 \\
0 & 1 & 0
\end{array}\right), \varphi_{3}=\left(\begin{array}{ccc}
0 & 1 & 0 \\
1 & 0 & 0 \\
0 & 0 & 0
\end{array}\right) \text {. }
$$




$$
\begin{gathered}
\xi_{1}=\left(\begin{array}{l}
0 \\
1 \\
0
\end{array}\right), \xi_{2}=\left(\begin{array}{l}
1 \\
0 \\
0
\end{array}\right), \xi_{3}=\left(\begin{array}{l}
0 \\
0 \\
1
\end{array}\right) . \\
\eta_{1}=(0,1,0), \eta_{2}=(1,0,0), \eta_{3}=(0,0,1) .
\end{gathered}
$$

The associated Riemannian metric being $g=\left(\begin{array}{lll}1 & 0 & 0 \\ 0 & 1 & 0 \\ 0 & 0 & 1\end{array}\right)$.

Note that, recently, other geometers have been interested by the problem of mixing paracontact and contact structures. This is the case of Mixed 3-Sasakian structures studied by [4] and Bi-paracontact structures studied by [5] as the paper of V. Martin-Molina [15].

For each $i$, the fundamental local 2-form, $\Phi_{i}$, is defined by

$$
\Phi_{i}(D, E)=g\left(D, \varphi_{i} E\right) .
$$

An almost paracontact manifold with 3-structure is called:

(1) 3-almost para-cosymplectic if $d \Phi_{i}=0=d \eta_{i}$;

(2) 3-para-cosymplectic if $\nabla \Phi_{i}=0$ and $\nabla \eta_{i}=0$;

(3) 3-para-Sasakian if $\Phi_{i}=d \eta_{i}$ and $N_{i}^{(1)}=0$, where $N_{i}^{(1)}$ designates the Nijenhuis tensor.

\section{Almost Paracontact 3-Submersions}

\subsection{The structure of the fibres}

By a Riemannian submersion, one understands a submersion

$$
\pi: M \longrightarrow M^{\prime}
$$

between Riemannian manifolds such that $\pi_{* \mid\left(K e r \pi_{*}\right)^{\perp}}$ is a linear isometry [16]. The tangent bundle $T(M)$ of the total space $M$ admits an orthogonal decomposition

$$
T(M)=V(M) \oplus H(M)
$$

where $V(M)$ and $H(M)$ denote, respectively, the vertical and the horizontal distribution. We denote by $\mathcal{V}$ and $\mathcal{H}$ the vertical and the horizontal projections of $T(M)$ onto $V(M)$ and $H(M)$ respectively. For all points $x^{\prime} \in M^{\prime}$, the closed embedded submanifold $F_{x^{\prime}}=\pi^{-1}\left(x^{\prime}\right)$ is called the fibre of $\pi$ over $x^{\prime}$. It is known that $\operatorname{dim} F_{x^{\prime}}=\operatorname{dim} M-\operatorname{dim} M^{\prime}$.

A vector field $X$ of the horizontal distribution is called a basic vector field if it is $\pi$-related to a vector field $X_{*}$ of the base space $M^{\prime}$. That is $X_{*}=\pi_{*} X$. On the base space, tensors and other operators will be specified by a prime 
$\left({ }^{\prime}\right)$ while those of the fibres will be denoted by a caret $\hat{()}$. For instance, $\nabla, \nabla^{\prime}$, and $\hat{\nabla}$ will designate the Levi-Civita connection of the total space, the base space and the fibres respectively.

Here, we put the definitions and some properties of the two types of submersions which will be considered.

Definition 3.1. Let $\left(M^{4 m+3}, g,\left(\varphi_{i}, \xi_{i}, \eta_{i}\right)_{i=1}^{3}\right)$ be an almost paracontact manifold with 3 -structure and $\left(M^{4 m^{\prime}}, g^{\prime},\left(J_{i}^{\prime}\right)_{i=1}^{3}\right)$ be an almost hyper-para-Hermitian manifold. A Riemannian submersion

$$
M^{4 m+3} \longrightarrow M^{\prime 4 m^{\prime}}
$$

is called an almost paracontact 3-submersion of type I, if it satisfies

$$
\pi_{*} \varphi_{i}=J_{i}^{\prime} \pi_{*}, i=1,2,3
$$

Proposition 3.2. Let $\pi: M^{4 m+3} \longrightarrow M^{\prime 4 m^{\prime}}$ be an almost paracontact 3-submersion of type I. Then the fibre submanifolds are invariant almost paracontact 3-manifolds.

Proof. Clearly the fibres are $4\left(m-m^{\prime}\right)+3$-dimensional submanifolds. Let $\left(g, \varphi_{i}, \xi_{i}, \eta_{i}\right)$ be the almost paracontact metric 3 -structure of the total space; note by $\left(\hat{g}, \hat{\varphi}_{i}, \hat{\xi}_{i}, \hat{\eta}_{i}\right)$ its restriction on the fibres. The problem is to show that $\left(\hat{g}, \hat{\varphi}_{i}, \hat{\xi}_{i}, \hat{\eta}_{i}\right)$ is an almost paracontact metric 3 -structure. Indeed, let $U$ and $V$ be two vertical vector fields tangent to the fibres. We have

$$
\begin{aligned}
& \hat{\varphi}_{i}^{2} U=U-\hat{\eta}_{i}(U) \hat{\xi}_{i} ; \\
& \hat{\eta}_{i}\left(\hat{\xi}_{i}\right)=\hat{g}\left(\hat{\xi}_{i}, \hat{\xi}_{i}\right)=g\left(\xi_{i}, \xi_{i}\right)=1 ; \\
& \hat{g}\left(\hat{\varphi}_{i} U, \hat{\varphi}_{i} V\right)=-\hat{g}(U, V)+\hat{\eta}_{i}(U) \hat{\eta}_{i}(V) .
\end{aligned}
$$

Surely the fibres are $\hat{\varphi}_{i}$-invariant. By definition, it is known that $\pi_{*} \hat{\varphi}_{i} U=J_{i}^{\prime} \pi_{*} U$. Let us denote by $k e r \pi_{*}$ the fibres; taking $U \in k e r \pi_{*}$, then $\pi_{*} U=0=\pi_{*} \hat{\varphi}_{i} U$ which means that $\hat{\varphi}_{i} U \in k e r \pi_{*}$.

Now, we are going to introduce a new type of almost paracontact 3-submersions. This type is closely related to almost contact 3-submersions of type II [18].

Definition 3.3. Let $\left(M^{4 m+3}, g,\left(\varphi_{i}, \xi_{i}, \eta_{i}\right)_{i=1}^{3}\right)$ and $\left(M^{\prime 4 m^{\prime}+3}, g^{\prime},\left(\varphi^{\prime}{ }_{i}, \xi^{\prime}{ }_{i}, \eta^{\prime}{ }_{i}\right)_{i=1}^{3}\right)$ two almost paracontact manifolds with

3-structure. A Riemannian submersion

$$
\pi: M^{4 m+3} \longrightarrow M^{\prime 4 m^{\prime}+3}
$$

is said to be an almost paracontact 3-submersion of type II if :

(a) $\pi_{*} \varphi_{i}=\varphi_{i}^{\prime} \pi_{*}$

(b) $\pi_{*} \xi_{i}=\xi_{i}^{\prime}$.

Proposition 3.4. Let $\pi: M^{4 m+3} \longrightarrow M^{\prime 4 m^{\prime}+3}$ be an almost paracontact 3 -submersion of type II. Then the fibres are almost hyper-para-Hermitian manifolds. 
Proof. Denoting by $F$ the fibres, it is clear that $\operatorname{dim} F=4\left(m-m^{\prime}\right)=4 n$ where $n=m-m^{\prime}$. On $\left(F^{4 n}, g\right)$, setting $J_{i}=\hat{\varphi}_{i}$,we have to show that $\left(g, J_{i}\right)$ is an almost para-Hermitian structure. Indeed,

$$
J_{i}^{2} U=\varphi_{i}^{2} U=U-\eta_{i}(U) \xi_{i}
$$

since $\eta_{i}(U)=0$ we have $J_{i}^{2} U=U$. On the other hand,

$$
g\left(J_{i} U, J_{i} V\right)=-g\left(U, J_{i}^{2} V\right)=-g(U, V)
$$

which achieves the proof.

\subsection{Transference of Structures}

The aim of this subsection is to determine the structure of the fibres and the base space according to that of the total space.

Proposition 3.5. Let $\pi: M^{4 m+3} \longrightarrow M^{\prime 4 m^{\prime}}$ be an almost paracontact 3-submersion of type I. If the total space is 3-para-cosymplectic or 3-para-Sasakian, then

(a) the base space is hyper-para-Kähler;

(b) the fibres inherit the structure of the total space.

Proof. First, we note that the manifolds under consideration have in common the following defining relations

$$
d \Phi_{i}(D, E, G)=0
$$

and

$$
N_{i}^{(1)}=0
$$

(a) Let $X, Y$ and $Z$ be basic vector fields. It is not hard to see that $\pi^{*} \Omega_{i}^{\prime}=\Phi_{i}$ from which we deduce $\pi^{*} d \Omega_{i}^{\prime}=d \Phi_{i}$. One can also establish that $\pi_{*} N_{i}^{(1)}=N_{i}^{\prime}$; since $d \Phi_{i}(X, Y, Z)=0$ and $N_{i}^{(1)}=0$, we have $\pi^{*} d \Omega_{i}^{\prime}=0=N_{i}^{\prime}$; therefore $d \Omega_{i}^{\prime}=0=N_{i}^{\prime}$ which define a 3 -para-Kähler structure on the base space for $\mathrm{i}=1,2,3$.

(b) It is well known that the vertical distribution is invariant by $\varphi_{i}$. Since $\eta_{i}(U)=\hat{\eta}_{i}(U)$ and

$$
\hat{N}_{i}^{(1)}(U, V)=N_{i}^{(1)}(U, V)
$$

we get

$$
d \hat{\Phi}_{i}(U, V, W)=0
$$

and $\hat{N_{i}^{(1)}}=0=d \hat{\eta}_{i}$.

Proposition 3.6. Let $\pi: M^{4 m+3} \longrightarrow M^{4 m^{\prime}}$ be an almost paracontact 3-submersion of type I. If the total space is 3-almost para-cosymplectic manifold, then

(a) the base space is hyper-para-almost Kähler, 
(b) the fibres inherit the structure of the total space.

Proof. As in the preceding Proposition 3.5, the manifold under consideration verifies the relation

$$
d \Phi_{i}(X, Y, Z)=0
$$

One can easily show that $d \Omega_{i}^{\prime}=0$ which is the defining relation of a 3-para-almost Kähler structure on the base space for $\mathrm{i}=1,2,3$. Accordingly, the base space is hyper-para-almost Kähler.

The case of the structure of the fibres is treated as in the preceding Proposition 3.5.

Now, we want to examine the structure of the fibres of a type II almost paracontact 3-submersion.

Proposition 3.7. Let $\pi: M^{4 m+3} \longrightarrow M^{4 m^{\prime}+3}$ be an almost paracontact 3 -submersion of type II. If the total space is 3-para-cosymplectic, then the fibres are hyper-para-Kähler.

Proof. The manifold under consideration verifies the following relations

$$
d \Phi_{i}(D, E, G)=0
$$

and

$$
N_{i}^{(1)}=0
$$

Thus $d \Phi_{i}(U, V, W)=0$. On the other hand, $\hat{N}_{i}(U, V)=0=N_{i}^{(1)}(U, V)$. Therefore, the fibres are defined by $d \hat{\Phi}_{i}=0=\hat{N}_{i}$ which are the defining relations of the hyper-para-Kähler structure.

Proposition 3.8. Let $\pi: M^{4 m+3} \longrightarrow M^{4 m^{\prime}+3}$ be an almost paracontact submersion of type II. Then the total space cannot be 3-para-Sasakian.

Proof. By absurd, suppose that the total space is a 3-para-Sasakian manifold and let us describe the structure of the fibres.

Recall from Proposition 3.4 that the fibres are almost hyper-para-Hermitian. Consider $U$ and $V$ two vertical vector fields tangent to the fibres. Since the total space is supposed to be 3 -para-Sasakian, it is defined by $\Phi_{i}=d \eta_{i}$ and $N_{i}^{(1)}=0$. Then $\Phi_{i}(U, V)=d \eta_{i}(U, V)$. But it can be shown that $d \eta_{i}(U, V)=0$ which implies that on the fibres, the fundamental local $2-$ form $\Omega_{i}(U, V)=0$. This is to say that, the fibres have the fundamental local $2-$ form zero for all $i=1,2,3$. Which is surely a non sense. Thus, the total space cannot be a 3 -para-Sasakian manifold.

\subsection{The Geometry of the Fibres}

We want to determine the classes of submersions with minimal or totally geodesic fibres. Let us recall that, on the total space of a Riemnnian submersion, B. O'Neill [16] has defined two configuration tensors, $T$ and $A$ of type (1,2) by setting

$$
T_{D} E=\mathcal{H} \nabla_{\mathcal{V} D} \mathcal{V} E+\mathcal{V} \nabla_{\mathcal{V} D} \mathcal{H} E
$$




$$
A_{D} E=\mathcal{V} \nabla_{\mathcal{H} D} \mathcal{H} E+\mathcal{V} \nabla_{\mathcal{H} D} \mathcal{V} E
$$

We shall be interested with the tensor $T$ which is a usefull tool in the study of the fibres. The tensor $A$ is used to measure the integrability of the horizontal distribution.

Note that the study of the $\varphi_{i}$-linearity of these tensors, in [17], is with helpfull. Recall that if $T_{\varphi_{i} U} V=\varphi_{i} T_{U} V$, then the fibres are minimal and if $T \equiv 0$ they are totally geodesic.

Lemma 3.9. Let $\pi: M^{4 m+3} \longrightarrow M^{\prime 4 m^{\prime}}$ be an almost paracontact 3-submersion of type I. If, for all $U$, $V$ and all $i=1,2,3, T_{U} V-T_{\varphi_{i} U} \varphi_{i} V=0$, then $T=0$.

Proof. It is an adaptation of [25, Lm3.4].

Corollary 3.10. Let $\pi: M^{4 m+3} \longrightarrow M^{4 m^{\prime}}$ be an almost paracontact 3-submersion of type I. If

$$
T_{U} \varphi_{i} V=\varphi_{i} T_{U} V
$$

then $T=0$.

Proof. If $T_{U} \varphi_{i} V=\varphi_{i} T_{U} V$, then $T_{\varphi_{i} U} \varphi_{i} V=\varphi_{i} T_{\varphi_{i} U} V$. Since $\varphi_{i} U$ is vertical and from the symmetric property of $T$ on vertical vector fields, we deduce

$$
T_{\varphi_{i} U} V=T_{V} \varphi_{i} U=\varphi_{i} T_{V} U=\varphi_{i} T_{U} V
$$

It is clear that

$$
T_{\varphi_{i} U} \varphi_{i} V=\varphi_{i}^{2} T_{U} V=T_{U} V-\eta_{i}\left(T_{U} V\right) \xi_{i}
$$

On the other hand, it can be shown that $T_{U} \xi_{i}=0$ and then

$\eta_{i}\left(T_{U} V\right)=0$ which gives $T_{\varphi_{i} U} \varphi_{i} V=T_{U} V$ from which $T_{U} V-T_{\varphi_{i} U} \varphi_{i} V=0$ follows. Applying Lemma 3.9, we get $T=0$.

Proposition 3.11. Let $\pi: M^{4 m+3} \longrightarrow M^{4 m^{\prime}}$ be an almost paracontact 3-submersion of type I. If the total space is 3-almost para-cosymplectic, then the fibres are totally geodesic.

Proof. It can be shown that the manifold under consideration satisfies the preceding Corrollary 3.10. The vanishing of $T$ is the condition for the fibres to be totally geodesic.

\subsection{Sectional Curvature Tensor}

Here, we want to determine the classes of almost paracontact 3 -submersions which preserve the $\varphi_{i}$-holomorphic sectional curvature tensor on horizontal or vertical distribution.

Proposition 3.12. Let $\pi: M^{4 m+3} \longrightarrow M^{\prime 4 m^{\prime}}$ be an almost paracontact 3-submersion of type I. Then the $\varphi_{i}{ }^{-}$ holomorphic sectional curvature tensor is defined by

(a) $H \varphi_{i}(U)=H \hat{\varphi}_{i}(U)+\|U\|^{-4}\left\{\left\|T_{U} \varphi_{i} U\right\|^{2}-g\left(T_{U} U, T_{\varphi_{i} U} \varphi_{i} U\right)\right\}$ 
(b) $H \varphi_{i}(X)=H_{J_{i}^{\prime}}\left(X_{*}\right)-3\|X\|^{-4}\left\|A_{X} \varphi_{i} X\right\|^{2}$.

Proof. Similar to that in [26, Thm.5.1(iv) and (v)].

Proposition 3.13. Let $\pi: M^{4 m+3} \longrightarrow M^{\prime 4 m^{\prime}}$ be an almost paracontact 3-submersion of type I. If the total space is 3-almost para-cosymplectic, then $H_{\varphi_{i}}(U)=H \hat{\varphi}_{i}(U)$ and $H_{\varphi_{i}}(X)=H_{J_{i}^{\prime}}\left(X_{*}\right)$.

Proof. From Proposition 3.11, we have $T=0$ which implies that

$H_{\varphi_{i}}(U)=H_{\hat{\varphi}_{i}}(U)$. Obviously, this manifold satisfies $A_{\varphi_{i} X} Y=\varphi_{i} A_{X} Y$ which gives rise to $A_{X} \varphi_{i} X=0$ and leads to $H_{\varphi_{i}}(X)=H_{J_{i}^{\prime}}\left(X_{*}\right)$.

\subsection{Some Examples}

In order to construct an example of a type I submersion, we will consider a manifold product as follows.

Let $\left(M^{\prime 4 m^{\prime}}, g^{\prime},\left(J_{i}^{\prime}\right)_{i=1}^{3}\right)$ be an almost hyper-para-Hermitian manifold and $\left(M^{4 m+3}, g,\left(\varphi_{i}, \xi_{i}, \eta_{i}\right)_{i=1}^{3}\right)$ an almost paracontact manifold

with 3 -structure. It is clear that the manifold product $\bar{M}=M^{\prime} \times M$ is of dimension $4\left(m^{\prime}+m\right)+3$ which will be taken $4 p+3$ where $p=m^{\prime}+m$. We can define a 3 -structure on $\bar{M}$ by setting

$$
\bar{\varphi}_{i}=\left(\begin{array}{cc}
J_{i} & 0 \\
0 & \varphi_{i}
\end{array}\right), \bar{\xi}_{i}=\frac{p}{m}\left(\begin{array}{c}
0 \\
\xi_{i}
\end{array}\right) \text { and } \bar{\eta}_{i}=\frac{m}{p}\left(0, \eta_{i}\right)
$$

It is not hard to show that $\left(\bar{M}, \bar{g},\left(\bar{\varphi}_{i}, \bar{\xi}_{i}, \bar{\eta}_{i}\right)_{i=1}^{3}\right)$ is an almost paracontact manifold with 3 -structure where $\bar{g}$ is defined by

$$
\bar{g}\left(\left(D^{\prime}, D\right),\left(E^{\prime}, E\right)\right)=g^{\prime}\left(D^{\prime}, E^{\prime}\right)+\frac{p^{2}}{m^{2}} g(D, E)
$$

We can refer to [23], where the product of paracontact manifolds is treated.

Proposition 3.14. Let $\left(M^{\prime 4 m^{\prime}}, g^{\prime},\left(J_{i}^{\prime}\right)_{i=1}^{3}\right)$ be an almost hyper-para-Hermitian manifold and $\left(M^{4 m+3}, g,\left(\varphi_{i}, \xi_{i}, \eta_{i}\right)_{i=1}^{3}\right)$ an almost paracontact manifold with 3 -structure. If $\left(M^{\prime} \times M, \bar{g},\left(\bar{\varphi}_{i}, \bar{\xi}_{i}, \bar{\eta}_{i}\right)_{i=1}^{3}\right)$ is an almost paracontact manifold with 3-structure, constructed as above, then it is 3-para-cosymplectic if, and only if, $M$ is 3-para-cosymplectic and $M^{\prime}$ is hyper-para-Kähler;

Proof. Let us note that, in this product, the calculation gives the following relation

$$
\left(\bar{\nabla}_{\left(D, r_{1} \frac{d}{d t}\right)} \bar{J}_{i}\right)\left(E, r_{2} \frac{d}{d t}\right)=\left(\left(\nabla_{D} \varphi_{i}\right) E-r_{2} \nabla_{D} \varphi_{i},\left(\nabla_{D} \eta_{i}\right) E \frac{d}{d t}\right)
$$

Suppose that $\left(g,\left(\varphi_{i}, \xi_{i}, \eta_{i}\right)_{i=1}^{3}\right)$ is a 3 -para-cosymplectic structure. It is known that in this case $\left(\nabla_{D} \eta_{i}\right)=0$ from which (3.1) gives

$$
\left(\bar{\nabla}_{\left(D, r_{1} \frac{d}{d t}\right)} \bar{J}_{i}\right)\left(E, r_{2} \frac{d}{d t}\right)=0
$$


which is the defining relation of a hyper-para-Kähler structure.

Conversely, if $\left(\bar{M}^{4 m}, \bar{g},\left(J_{i}^{\prime}\right)_{i=1}^{3}\right)$ is hyper-para-Kählerian manifold, then in (3.1) we have

$$
\left(\nabla_{D} \varphi_{i}\right) E-r_{2} \nabla_{D} \varphi_{i}=0
$$

or

$$
\left(\nabla_{D} \eta_{i}\right) E=0
$$

But each of these two properties characterizes the 3-para-cosymplectic structure.

Now, we have

Proposition 3.15. Let $\bar{M}=M^{\prime} \times M$ be the manifold product constructed as above, then the projection

$$
\pi: M^{\prime} \times M \longrightarrow M^{\prime}
$$

is an almost paracontact 3-submersion of type $I$.

Proof. The problem is to show that $\pi_{*} \bar{\varphi}_{i}=J_{i}^{\prime} \pi_{*}$. Indeed,

$\bar{\varphi}_{i}\left(D^{\prime}, D\right)=\left(J_{i}^{\prime} D^{\prime}, \varphi_{i} D\right)$ implies that

$$
\pi_{*} \bar{\varphi}_{i}\left(D^{\prime}, D\right)=\pi_{*}\left(J_{i}^{\prime} D^{\prime}, \varphi_{i} D\right)=J_{i}^{\prime} D^{\prime}
$$

On the other hand, $\pi_{*}\left(D^{\prime}, D\right)=D^{\prime}$ and so, $J_{i}^{\prime} \pi_{*}\left(D^{\prime}, D\right)=J_{i}^{\prime} D^{\prime}$ which gives rise to

$$
\pi_{*} \bar{\varphi}_{i}\left(D^{\prime}, D\right)=J_{i}^{\prime} D^{\prime}=J_{i}^{\prime} \pi_{*}\left(D^{\prime}, D\right)
$$

from which $\pi_{*} \bar{\varphi}_{i}=J_{i}^{\prime} \pi_{*}$ follows.

Since $\bar{\varphi}_{i}(D, 0)=\left(J_{i}^{\prime} D^{\prime}, 0\right)$ and $\bar{\varphi}_{i}(0, D)=\left(0, \varphi_{i} D\right)$, then $M$ is an invariant submanifold of $M^{\prime} \times M$ by $\varphi_{i}$.

\section{References}

[1] Alekseevsky, D.V. and Kamishima, Y.: Quaternionic and para-quaternionic CR structures on (4n+3) dimensional manifolds, Central European J. Math. 2 (5) (2004),732-753.

[2] Cabrera, F.M. Almost hyper-Hermitian structures in bundles spaces over manifolds with almost contact 3-structure, Czech. Math. J. 48 (3) (1998),545-563.

[3] Caldarella, A.V., On paraquaternionic submersions between paraquaternionic Kähler manifolds, Acta Math., 112 (2010),1-14.

[4] Caldarella, A.V., and Pastore, A.M.,Mixed 3-Sasakian structures and curvature, Ann. Polon. Math., 96(2) (2009),107-125.

[5] Cappelletti-Montano, B., Bi-paracontact structures and Legendre foliations, Kodai Math. J., 33(2010),473512. 
[6] Cappelletti-Montano, B., Carriazo, A. and Martin-Molina, V., Sasaki-Einstein and paraSasaki-Einstein metrics from $(k, \mu)$-structures, J. Geom. Phys., 73 (2013),20-36.

[7] DAcko, P., On almost para-cosymplectic manifolds, Tsukuba J. Math. 28 (2004),193-213.

[8] Dacko, P. and Olszak, Z. On weakly para-cosymplectic manifolds of dimension 3, J. Geom. Physics, 57 (2007), 561-570.

[9] Deshmukh, S. and Khan, A.G., Almost paracontact 3-structure on a differentiable manifold, Indian J. pure appl. Math., 10 (4)(1979), 442-448.

[10] Gunduzalp, Y. and Sahin, B., Paracontact semi-Riemannian submersions, Turkish J. Math., 37 (2013), $114-128$.

[11] Ivanov, S. and Zamkovoy, S. Para-Hermitian and para-quaternionic manifolds, Differential Geom. Appl., 23 (2005),205-234.

[12] Kaneyuki, S. and Williams, F.L., Almost paracontact and paraHodge structures on manifolds, Nagoya Math. J., 99 (1985), 173-187.

[13] Kupeli Erken, I., On normal almost paracontact metric manifolds of dimension 3, Facta Universitatis (Nis) Ser. Math. Inform. 30 (5) (2015), 777-788.

[14] Kupeli Erken, I., Some clases of 3-dimensional normal almost paracontact metric manifolds, Honam Mathematical J. 37 (4) (2015) 457-468.

[15] Martin-Molina, V., Paracontact metric manifolds without a contact metric counterpart, Taiwanese J. Math., 19 (2015), 175-191.

[16] O'NeILl, B., The fundamental equations of a submersion, Michigan Math.J., 13 (1966), 459-469.

[17] Tshikuna-Matamba, T.: On the $\varphi$-linearity of the configuration tensors of an almost contact metric submersion, Bull. Math. Soc. Sci. Math. de Roumanie,37 (1993),165-174.

[18] Tshikuna-Matamba, T.: Geometric properties of almost contact metric 3-submersions, Periodica Math. Hungar, 52(1) (2006),101-119.

[19] Tshikuna-Matamba, T., On the fibres of an almost paracontact metric submersion, J. Math. Univ. Tokushima, 50 (2016), 1-13.

[20] Tshikuna-Matamba, T., On the base space of an almost paracontact submersion, Facta Universitatis (Nis), Ser. Math. Inform.,31 (5) (2016), 1041-1049.

[21] Tshikuna-Matamba, T., Some properties of $G_{1}$-paracontact submersions, Global Journal of Mathematics, 9 (2) (2017),585-594.

[22] Tshikuna-Matamba, T., On $G_{2}$-paracontact submersions, Journal of Advance in Mathematical Science, 3 (2) (2017),224-237.

[23] Tshikuna-Matamba, T., Produit de variétés en géométrie paracontacte, Ann.Sc. Unikin, 2 (2017),1-8.

[24] Tshikuna-Matamba, T., Induced Structures on the Product of Manifolds in Paracontact Geometry, Global J. Math, 14(1) (2019),922-930. 
[25] Watson, B.: G,G'-Riemannian submersions and non linear gauge field equations of general relativity, Global Analysis-Analysis on manifolds, Ed.T.M. Rassias, Teubner Press, Leipzig ,1983,pp.324-349.

[26] Watson, B.: Almost contact metric 3-submersions, Int.J.Math.Math. Sci.,7 (1984),667-688.

[27] Watson, B.: The differential geometry of two types of almost contact metric submersions, The Math. Heritage of C.F. Gauss, Ed. G.M. Rassias, World Sci. Pub.Co., Singapore,1991,pp.827-861.

[28] Zamkovoy, S., Cannonical connections on paracontact manifolds, Ann. Global Anal. Geometry, 36 (2009),37-60.

Département de Mathématiques Université Pédagogique de Kananga B.P. 282-Kananga République Démocratique du Congo e-mail address: tshikmat@gmail.com 